\title{
Surgical airway procedures in emergency surgical patients: Results of what has become a back-up procedure
}

\author{
Gijs J. A. Willinge ${ }^{1,2}$ (D) Falco Hietbrink ${ }^{1} \cdot$ Luke P. H. Leenen $^{1}$
}

Accepted: 31 March 2021/Published online: 22 May 2021

(c) The Author(s) 2021

\begin{abstract}
Background Cricothyroidotomy and surgical tracheostomy are methods to secure airway patency. In emergency surgery, these methods are nowadays mostly reserved for patients unsuited for percutaneous procedures. Detailed description of complications and functional outcomes following both procedures is underreported in current literature. The aim of this study was to evaluate outcomes following cricothyroidotomy and tracheostomy in this presumed complex population.

Methods In this retrospective cohort study, adult emergency surgical patients treated with cricothyroidotomy and/or surgical tracheostomy were included. Postoperative complications and functional outcomes in trauma and nontrauma patients were evaluated.

Results Forty-one trauma patients and 11 non-trauma emergency surgical patients (mainly after elective oncoabdominal or vascular surgery) were included. Of 52 patients, seven underwent cricothyroidotomy pre-tracheostomy. Mortality was higher in non-trauma patients $(p=0.04)$ following both procedures. Over half of patients $(56 \%$, $n=29$ ) regained unsupported airway patency with a tendency toward increased tracheostomy removal in trauma patients. Among complications, only pneumonia occurred frequently $(60 \%, n=31)$, with no relation to patient type. Other complications included local infection $(5.8 \%, n=4)$ and wound dehiscence $(1.9 \%, n=1)$. Adverse functional outcomes were frequently observed and were mild and self-limiting. Cervical spinal cord injury reduced overall unsupported airway patency $(p=0.01)$; with high cervical spinal cord injury related to adverse functional outcomes and increased home ventilation need.

Conclusions No major procedure-related complications or functional adverse events were encountered following cricothyroidotomy and surgical tracheostomy, even though only complex patients were included. Only mild, selflimiting functional problems occurred, especially in trauma patients with cervical injury who underwent early tracheostomy by longitudinal incision. This information can aid clinicians in making tailor-made decisions for individual patients.
\end{abstract}

Gijs J. A. Willinge

gijs_willinge@hotmail.com

Falco Hietbrink

F.Hietbrink@umcutrecht.nl

Luke P. H. Leenen

L.P.H.Leenen@umcutrecht.nl
1 Department of Traumatology, University Medical Center Utrecht, Utrecht, The Netherlands

2 Utrecht Trauma Center, G04. 228, Heidelberglaan 100, 3584 CX Utrecht, The Netherlands 


\section{Introduction}

Cricothyroidotomy and tracheostomy are both surgical methods to secure patency of the airway. Although serving the same purpose, both procedures are performed in different settings and have different indications. Cricothyroidotomy is performed in the emergency setting and provides an alternative method of gaining airway access when endotracheal intubation fails. Nowadays, with videoguided intubation techniques, this invasive procedure is rarely performed, but it can still be crucial in preventing anoxic encephalopathy and death. Considering the emergency setting, it is not surprising cricothyroidotomy is prone to cause severe short-term complications, including upper airway laceration, posterior tracheal perforation and nerve damage [1, 2]. Possibly due to its rare nature, knowledge about long-term outcomes following cricothyroidotomy is scarce.

Tracheostomy is a more common procedure in current medical practice [3]. Apart from its necessity during head\&neck surgery, it is often electively performed when the need for prolonged mechanical ventilation (MV) is expected. A tracheostomy provides a way to maintain airway patency for an increased period of time. It has proved to cause less laryngeal complications, improve patient comfort and pulmonary hygiene, and reduce the use of sedation compared to prolonged endotracheal intubation [4-7]. Furthermore, it enables faster weaning from MV by reducing airway resistance and decreasing dead ventilation space $[6,8]$. Two techniques for tracheostomy are currently used: percutaneous dilatational tracheostomy and surgical tracheostomy. The percutaneous method has gained preference over the surgical method in recent years due to suggested lower peri-operative complication rates and higher cost-effectiveness [9]. Surgical tracheostomy is now specifically used when contra-indications for percutaneous dilatational tracheostomy (such as history of neck surgery, unstable or immobile cervical spine, or anatomical anomalies) exist [10, 11]. Compared to cricothyroidotomy, tracheostomy causes less short-term complications. However, studies have described long-term complications and adverse functional outcomes following this procedure [12]. Although large database studies exist, detailed descriptions of these complications and functional outcomes are limited [13].

Improvement of detailed knowledge on consequences could assist surgeons in deciding whether to perform these invasive procedures, when to perform them and for which patient they would be beneficial. Therefore, the aim of this study was to analyze short- and long-term outcomes following both cricothyroidotomy and surgical tracheostomy in emergency surgical patients and to identify specific factors contributing to (relative) adverse outcomes.

\section{Materials and methods}

\section{Study design and population}

This was a single center, retrospective cohort study. A waiver of the Medical Ethical Committee of University Medical Center Utrecht (UMCU) was provided to conduct this study. Records of all emergency surgical patients receiving cricothyroidotomy and/or open surgical tracheostomy at the UMCU between January 2013 and December 2018 were identified. Emergency surgical patients aged $\geq 18$ years at time of surgery, receiving cricothyroidotomy and/or surgical tracheostomy, were included. Patients were excluded if they received an initial airway procedure elsewhere (except pre-hospital cricothyroidotomy with presentation at our center subsequently). This study focused solely on the open surgical tracheostomy technique and not the percutaneous technique. Subsequently, as surgical tracheotomy is considered a back-up procedure at our hospital and is only used in patients with contra-indications for percutaneous tracheostomy, only complex emergency surgery patients for tracheostomy placement were included. For the remainder of this article, 'tracheostomy' will refer to the surgical technique.

\section{Study treatment specifics}

Our center features an intermediate care unit (IMCU), which can provide hemodynamic monitoring and respiratory supportive care, and a fully equipped intensive care unit (ICU) [14]. Regarding cricothyroidotomy, standard procedure included conversion of cricothyroidotomy to tracheostomy within at least 14 days after cricothyroidotomy. This was generally done as soon as reasonably possible to avoid potential complications at the cricothyroidotomy surgery site, as recommended by current literature $[15,16]$. For all tracheostomy procedures, one of two incision types was performed: a longitudinal incision or a hatch-shaped incision. Choice of incision type was based on personal preferences of the operating surgeon. All procedures were performed or supervised by senior specialist level trauma surgeons.

\section{Data collection}

Relevant data were extracted from patient records. This included-but was not limited to-patient and injury characteristics, procedural characteristics, occurrence of 
postoperative complications at short- and long-term, physical airway-related complaints, surgical hardware-related complications, unsupported patency of airway, duration of weaning, duration of decuffing, time until removal of tracheostomy and mortality. Indications for tracheostomy were subdivided into three main groups: 1) physically threatened airway, 2) respiratory insufficient function (including ICU acquired weakness, neurological disability and prolonged/impaired weaning) and 3) alternative way of airway access (following cricothyroidotomy or endotracheal intubation complications). Included patients were divided into two groups for primary analysis: trauma patients and non-trauma patients. For trauma patients, injuries to the head/neck region were specified into head, brain, cervical osseous and cervical spinal cord injuries. Brain injury was additionally scored based on Glasgow Coma Scale (GCS) motor scores, with mild as GCS motor score 4-5 and severe as GCS motor score $\leq 3$.

\section{Outcome measures}

Primary outcome measures were airway-related complications at short- and long-term (up to a maximum of one year after the procedure) following both cricothyroidotomy and tracheostomy. These complications included lacerations of upper respiratory structures (such as vocal cords, pharynx and the tracheal wall), obstruction, posterior tracheal perforation, creation of false tracts, local infections, pneumothorax, wound dehiscence, pneumonia and mortality. Secondary outcomes were length of hospital stay, length of ICU stay, material-related complications and functional airway-related outcomes at short- and long-term follow-up (up to a maximum of one year after the procedure). This included phonation problems following tracheostomy, inability to autonomously clean airway, sputum management and swallowing dysfunction. Additionally, ability to wean, decuff and ultimately remove the tracheostomy was assessed. When applicable, duration of weaning, decuffing and time to removal of the tracheostomy was evaluated. To identify risk factors for adverse functional outcomes, patient and (if applicable) injury characteristics were analyzed.

\section{Statistical analysis}

Statistical analysis was performed using IBM SPSS (Statistics for Windows, Version 25.0. Armonk, NY: IBM Corp). Descriptive data of continuous variables were summarized using appropriate measures of central tendency (i.e., mean, median) and dispersion (i.e., standard deviation, interquartile range [IQR]), depending on the distribution of variables. Categorical variables were presented using frequency measures. The Pearson Chi-square test and Fisher Exact test (two-sided) were used to analyze statistical significance of relations between categorical variables. The independent t-test was used for continuous variables. A p-value $\leq 0.05$ indicated a statistically significant relation between variables.

\section{Results}

A total of 61 patients received cricothyroidotomy and/or tracheostomy of which 52 met inclusion criteria (Fig. 1). Of these 52 patients, 45 underwent isolated tracheostomy and seven received cricothyroidotomy before tracheostomy. All cricothyroidotomies were converted into a tracheostomy within three days. Of all included patients, 41 (79\%) were trauma patients. Median age was 54 years (IQR: 35) in the trauma group and 67 years (IQR: 13) in the non-trauma group, with a relatively low median age of cricothyroidotomy patients in both groups (Table 1). ASA score was higher in non-trauma patients compared to trauma patients. The non-trauma group consisted mainly of patients in need of surgical airway management after complications following elective abdominal or vascular surgery. Excessive sputum production pre-tracheostomy (reduced airway hygiene) was observed in 19 (37\%) patients.

Focusing on procedural specifics, prolonged insufficient respiratory function was the most common indication for tracheostomy and the longitudinal incision was most frequently performed. Indications for cricothyroidotomy included unsuccessful intubation $(n=5 ; 71 \%)$ and acute physically threatened airway $(n=2 ; 29 \%)$. Of the latter, one patient was successfully intubated after which the endotracheal tube was obstructed by active bleeding without successful exchange, and cricothyroidotomy was performed. The other patient was impossible to intubate due to facial injuries and therefore underwent primary cricothyroidotomy.

\section{Surgical outcomes}

Mortality within one year after tracheostomy was relatively high in non-trauma patients, with $45 \%(n=5)$ compared to $15 \%(n=6)$ in trauma patients $(p=0.04)$ (Table 2). Our data showed no direct link between cricothyroidotomy and/ or tracheostomy and the cause of death. For trauma patients, the primary cause of mortality was a direct consequence of the initial trauma, mainly neurological. For non-trauma patients, this was as a consequence of the underlying medical condition (e.g., sepsis, cancer, abdominal ischemia). Of trauma patients, 90\% $(n=37)$ was successfully weaned from MV compared to $55 \%$ $(n=6)$ of non-trauma patients $(p=0.01) .59 \%$ of trauma 


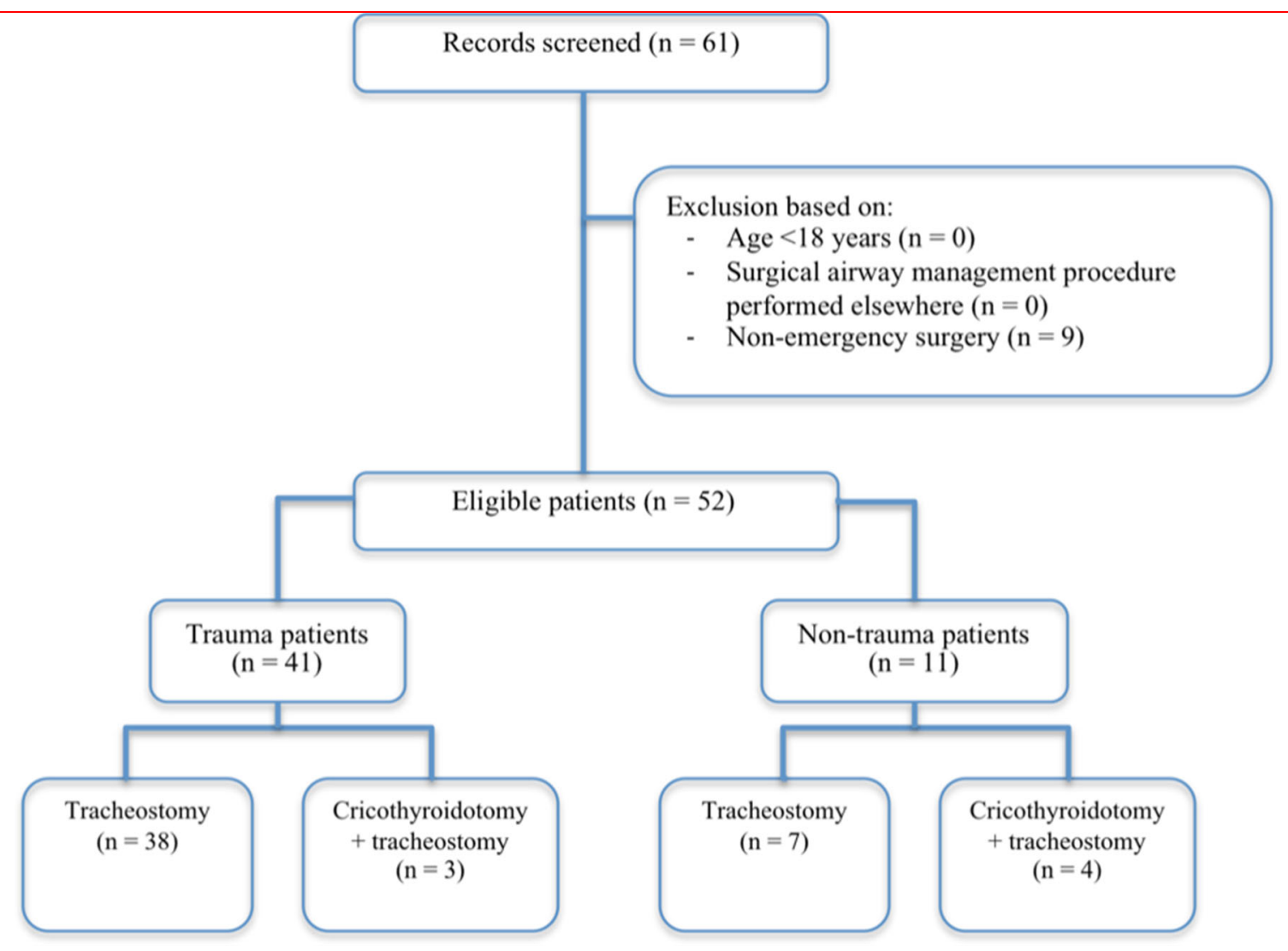

Fig. 1 Flowchart of patient selection for study inclusion

patients $(n=24)$ regained unsupported airway patency following both cricothyroidotomy and tracheostomy, compared to $45 \%(n=5)$ of non-trauma patients $(p=0.5)$. Length of ICU stay was longer in non-trauma patients (median 38 days, IQR 44) compared to trauma patients (median 16 days, IQR 13) $(p=0.05)$. Focusing on injury characteristics in trauma patients, cervical spinal cord injury was associated with decreased successful weaning rate $(p=0.01)$, decreased unsupported airway patency $(p=0.01)$ and less removal of tracheostomy $(p=0.01)$. No significant associations between these outcomes and other injury characteristics existed. Among complications, only pneumonia was frequently present in all patient groups $(60 \%, n=31)$, with no significant relation to specific patient type. Other procedure-related complications included local infection $(5.8 \%, n=4)$ and wound dehiscence $(1.9 \%, n=1)$.

\section{Functional outcomes}

Physical airway complaints (pain/irritation and swallowing dysfunction) predominantly occurred in trauma patients.
All complaints started within ten days after tracheostomy and disappeared within one year. Material-related complications were exclusively seen in trauma patients, with acquired cuff insufficiency being most common. It was unknown if these adverse functional outcomes (non-material-related) and complications were a direct consequence of the tracheostomy or were caused by trauma-related factors (such as neurological injuries or injuries to the maxillofacial or cervical region). Furthermore, spinal cord injury (level C1-C4) was significantly associated with phonation problems $(p=0.03)$ and inability to clean airway $(p=0.05)$ (Table 3$)$. Need for home ventilation support was also higher in these patients $(p<0.01)$. Of five patients with phonation problems after high cervical spinal cord injury, two had physical complaints during phonation after tracheostomy and three were unable to speak due to the nature of their injury.

For trauma patients, a significant relation existed between incision type and increased sputum leakage at tracheostomy site $(p=0.04)$. Furthermore, of patients with increased sputum leakage, $80 \%$ underwent early tracheostomy ( $\leq 14$ days) and $60 \%$ had cervical injuries. 
Table 1 Baseline characteristics

\begin{tabular}{|c|c|c|c|c|}
\hline \multirow{2}{*}{$\begin{array}{l}\text { Variables } \\
\text { Demographics }\end{array}$} & \multicolumn{2}{|l|}{ Tracheostomy } & \multicolumn{2}{|c|}{ Cricothyroidotomy + tracheostomy } \\
\hline & $\begin{array}{l}\text { Trauma } \\
N=38 \\
\text { Median (IQR) }\end{array}$ & $\begin{array}{l}\text { Non-trauma } \\
N=7 \\
\text { Median (IQR) }\end{array}$ & $\begin{array}{l}\text { Trauma } \\
N=3 \\
\text { Median (IQR) }\end{array}$ & $\begin{array}{l}\text { Non-trauma } \\
N=4 \\
\text { Median (IQR) }\end{array}$ \\
\hline Age (years) & $55(37)$ & $67(9)$ & $39 * *$ & $62(24)$ \\
\hline BMI & $24(9)$ & $27(6)$ & $25^{* *}$ & $28(11)$ \\
\hline \multirow[t]{2}{*}{ Variables } & \multicolumn{2}{|l|}{ Tracheostomy } & \multicolumn{2}{|c|}{ Cricothyroidotomy + tracheostomy } \\
\hline & $\begin{array}{l}\text { Trauma } \\
N=38 \\
N(\%)\end{array}$ & $\begin{array}{l}\text { Non-trauma } \\
N=7 \\
N(\%)\end{array}$ & $\begin{array}{l}\text { Trauma } \\
N=3 \\
N(\%)\end{array}$ & $\begin{array}{l}\text { Non-trauma } \\
N=4 \\
N(\%)\end{array}$ \\
\hline Male & $25(66)$ & $4(57)$ & $3(100)$ & $1(25)$ \\
\hline Diabetes mellitus & $4(11)$ & $1(14)$ & $0(0)$ & $0(0)$ \\
\hline \multicolumn{5}{|l|}{ ASA score } \\
\hline 1 & $0(0)$ & $0(0)$ & $0(0)$ & $0(0)$ \\
\hline 2 & $23(61)$ & $1(14)$ & $2(67)$ & $0(0)$ \\
\hline 3 & $15(40)$ & $6(86)$ & $1(33)$ & $3(75)$ \\
\hline 4 & $0(0)$ & $0(0)$ & $0(0)$ & $1(25)$ \\
\hline \multicolumn{5}{|l|}{ Cervical characteristics } \\
\hline Obesity & $7(18)$ & $1(14)$ & $0(0)$ & $1(25)$ \\
\hline Anatomical abnormalities & $4(11)$ & $0(0)$ & $0(0)$ & $0(0)$ \\
\hline Surgical history in cervical region & $1(3)$ & $1(14)$ & $0(0)$ & $0(0)$ \\
\hline Pre-tracheostomy swallowing dysfunction & $7(18)$ & $0(0)$ & $0(0)$ & $0(0)$ \\
\hline Excessive sputum pre-tracheostomy & $15(40)$ & $3(43)$ & $0(0)$ & $1(25)$ \\
\hline Variables & \multicolumn{2}{|l|}{ Tracheostomy } & \multicolumn{2}{|c|}{ Cricothyroidotomy + tracheostomy } \\
\hline Clinical characteristics & $\begin{array}{l}\text { Trauma } \\
N=38 \\
\text { Median (IQR) }\end{array}$ & $\begin{array}{l}\text { Non-trauma } \\
N=7 \\
\text { Median (IQR) }\end{array}$ & $\begin{array}{l}\text { Trauma } \\
N=3 \\
\text { Median (IQR) }\end{array}$ & $\begin{array}{l}\text { Non-trauma } \\
N=4 \\
\text { Median (IQR) }\end{array}$ \\
\hline ISS* & $27(15)$ & - & $22 * *$ & - \\
\hline \multicolumn{5}{|l|}{ Vital signs at day of tracheostomy } \\
\hline Systolic blood pressure & $120(35)$ & $120(38)$ & $90 * *$ & $132(17)$ \\
\hline Diastolic blood pressure & $70(20)$ & $67(30)$ & $75^{* *}$ & $64.8(12)$ \\
\hline Heart rate & $93(37)$ & $87(41)$ & $90 * *$ & $95(34)$ \\
\hline \multirow[t]{2}{*}{ Variables } & \multicolumn{2}{|l|}{ Tracheostomy } & \multicolumn{2}{|c|}{ Cricothyroidotomy + tracheostomy } \\
\hline & $\begin{array}{l}\text { Trauma } \\
N=38 \\
N(\%)\end{array}$ & $\begin{array}{l}\text { Non-trauma } \\
N=7 \\
N(\%)\end{array}$ & $\begin{array}{l}\text { Trauma } \\
N=3 \\
N(\%)\end{array}$ & $\begin{array}{l}\text { Non-trauma } \\
N=4 \\
N(\%)\end{array}$ \\
\hline ISS $>15^{*}$ & $32(84)$ & - & $2(67)$ & - \\
\hline \multicolumn{5}{|l|}{ GCS* } \\
\hline GCS motor $\leq 3$ & $18(47)$ & $1(14)$ & $1(33)$ & $2(25)$ \\
\hline $\mathrm{GCS} \leq 8$ & $20(53)$ & $1(14)$ & $1(33)$ & $2(25)$ \\
\hline ICU admission & $38(100)$ & $6(86)$ & $3(100)$ & $3(75$ \\
\hline IMCU admission & $0(0)$ & $1(14)$ & $0(0)$ & $1(25)$ \\
\hline \multicolumn{5}{|l|}{ Trauma injury specifics } \\
\hline Head injury & $23(61)$ & $0(0)$ & $1(33)$ & $0(0)$ \\
\hline \multicolumn{5}{|l|}{ Brain injury* } \\
\hline Mild (GCS motor 4-5) & $9(24)$ & $0(0)$ & $1(33)$ & $0(0)$ \\
\hline Severe $($ GCS motor $\leq 3)$ & $18(47)$ & $0(0)$ & $0(0)$ & $0(0)$ \\
\hline Cervical osseous injury & $19(50)$ & $0(0)$ & $2(67)$ & $0(0)$ \\
\hline
\end{tabular}


Table 1 continued

\begin{tabular}{|c|c|c|c|c|}
\hline \multirow[t]{3}{*}{ Variables } & \multicolumn{2}{|c|}{ Tracheostomy } & \multicolumn{2}{|c|}{ Cricothyroidotomy + tracheostomy } \\
\hline & \multirow{2}{*}{$\begin{array}{l}\text { Trauma } \\
N=38 \\
N(\%)\end{array}$} & \multirow{2}{*}{$\begin{array}{l}\text { Non-trauma } \\
N=7 \\
N(\%)\end{array}$} & \multirow{2}{*}{$\begin{array}{l}\text { Trauma } \\
N=3 \\
N(\%)\end{array}$} & \multirow{2}{*}{$\begin{array}{l}\text { Non-trauma } \\
N=4 \\
N(\%)\end{array}$} \\
\hline & & & & \\
\hline \multicolumn{5}{|l|}{ Cervical spinal cord injury } \\
\hline Level C1-C4 & $5(13)$ & $0(0)$ & $0(0)$ & $0(0)$ \\
\hline Level C5-C8 & $2(5)$ & $0(0)$ & $0(0)$ & $0(0)$ \\
\hline \multicolumn{5}{|l|}{ Procedural specifics } \\
\hline \multicolumn{5}{|c|}{ Time from trauma/initial surgery to tracheostomy } \\
\hline Early ( $\leq 14$ days) tracheostomy & $28(74)$ & $3(43)$ & $3(100)$ & $4(100)$ \\
\hline Late ( $>14$ days) tracheostomy & $10(27)$ & $4(57)$ & $0(0)$ & $0(0)$ \\
\hline \multicolumn{5}{|l|}{ Indication for tracheostomy } \\
\hline Physically threatened airway & $3(8)$ & $0(0)$ & $1(33)$ & $1(25)$ \\
\hline Insufficient respiratory function & $34(90)$ & $6(86)$ & $0(0)$ & $0(0)$ \\
\hline Alternative way of airway management & $1(3)$ & $1(14)$ & $2(67)$ & $3(75)$ \\
\hline \multicolumn{5}{|l|}{ Incision type* } \\
\hline Longitudinal & $26(69)$ & $2(29)$ & $2(67)$ & $2(50)$ \\
\hline U-shaped & $9(24)$ & $2(29)$ & $0(0)$ & $1(25)$ \\
\hline
\end{tabular}

$I Q R$ Interquartile range, $B M I$ Body Mass Index, ASA American society of Anesthesiologists, ISS Injury severity score, GCS Glasgow coma scale, $I C U$ Intensive care unit, IMCU Intermediate care unit

*Missing data: ISS was missing in $8 \%(n=3)$ of trauma tracheostomy patients. No ISS was scored in non-trauma patients. GCS was missing in $9 \%(n=4)$ of total tracheostomy patients; GCS was missing in 5\% $(n=2)$ of trauma tracheostomy patients and in 29\% ( $n=2)$ of non-trauma tracheostomy patients. GCS was missing in 2 of non-trauma cricothyroidotomy + tracheostomy patients. Incision type was missing in $15 \%$ $(n=7)$ of total tracheostomy patients; Incision type was missing in $8 \%(n=3)$ of trauma tracheostomy patients and in $43 \%(n=3)$ of nontrauma tracheostomy patients. Incision type was missing in $33 \%(n=1)$ of trauma cricothyroidotomy + tracheostomy patients and in $25 \%$ $(n=1)$ of non-trauma cricothyroidotomy + tracheostomy patients

**In several cases, IQR could not be presented due to group size of $n \leq 3$

There was no significant relation between sputum leakage after tracheostomy and inability to clean airway, or excessive sputum pre-tracheostomy. Inability to clean airway was more frequently seen in patients receiving early tracheostomy ( $\leq 14$ days after trauma/initial surgery).

\section{Discussion}

This study provided a detailed description of short- and long-term clinical outcomes following surgical airway procedures in emergency surgical patients, in a time where these procedures are reserved for patients unsuited for percutaneous procedures. Over half of patients regained unsupported airway patency, with a tendency toward increased removal of tracheostomy in trauma patients. As expected, cervical spinal cord injury was associated with reduced unsupported patency of airway. No procedure-related complications were encountered. Adverse functional outcomes did occur, but were considered mild and were all self-limiting.
Mortality rate after tracheostomy is high, emphasizing the dire situation of patients in need of cricothyroidotomy and/or tracheostomy $[17,18]$. In our study, overall mortality was $21 \%$, with mortality in non-trauma patients (45\%) even three times higher compared to trauma patients (15\%). This is possibly due to the medical condition of non-trauma patients before tracheostomy (as represented by ASA scores at baseline) and underlying disease, which initially caused their ICU admission. No direct link was found between surgical airway management procedures and cause of death. Patients predominantly died due to direct consequences of trauma (e.g., neurological damage) or due to complications related to the underlying medical condition/disease (e.g., abdominal ischemia, sepsis, cancer, complication of aortic repair). Although no link between surgical airway management and mortality existed, it can be stated that the sole need for surgical airway management, especially in non-trauma patients, is a harbinger of complications. Other studies similarly showed a higher mortality rate in patients with underlying (specifically respiratory) disease $[19,20]$. Additionally, regarding length 
Table 2 Comparison of outcomes between tracheostomy and cricothyroidotomy + tracheostomy in trauma and non-trauma patients

\begin{tabular}{|c|c|c|c|c|}
\hline \multirow[t]{2}{*}{ Variables } & \multicolumn{2}{|l|}{ Tracheostomy } & \multicolumn{2}{|c|}{ Cricothyroidotomy + tracheostomy } \\
\hline & $\begin{array}{l}\text { Trauma } \\
N=38 \\
N(\%)\end{array}$ & $\begin{array}{l}\text { Non-trauma } \\
N=7 \\
N(\%)\end{array}$ & $\begin{array}{l}\text { Trauma } \\
N=3 \\
N(\%)\end{array}$ & $\begin{array}{l}\text { Non-trauma } \\
N=4 \\
N(\%)\end{array}$ \\
\hline \multicolumn{5}{|l|}{ Complications } \\
\hline Upper airway lacerations & $0(0)$ & $0(0)$ & $0(0)$ & $0(0)$ \\
\hline Posterior tracheal perforation & $0(0)$ & $0(0)$ & $0(0)$ & $0(0)$ \\
\hline False tracts & $0(0)$ & $0(0)$ & $0(0)$ & $0(0)$ \\
\hline Pneumothorax & $0(0)$ & $0(0)$ & $0(0)$ & $0(0)$ \\
\hline Wound dehiscence & $1(3)$ & $0(0)$ & $0(0)$ & $0(0)$ \\
\hline Local infection & $2(5)$ & $0(0)$ & $0(0)$ & $1(25)$ \\
\hline Pneumonia & $24(63)$ & $3(43)$ & $1(33)$ & $3(75)$ \\
\hline Obstruction & $1(3)$ & $0(0)$ & $0(0)$ & $0(0)$ \\
\hline Mortality $<1$ year after tracheostomy & $6(16)$ & $4(57)$ & $\mathbf{0}(\mathbf{0})$ & $1(25)$ \\
\hline \multicolumn{5}{|l|}{ Tracheostomy outcomes } \\
\hline Successfully weaned & $34(90)$ & $3(43)$ & $3(100)$ & $3(75)$ \\
\hline Decuffing complete & $26(68)$ & $2(29)$ & $2(67)$ & $3(75)$ \\
\hline Removal of tracheostoma & $21(55)$ & $2(29)$ & $3(100)$ & $3(75)$ \\
\hline Overall unsupported airway patency & $21(55)$ & $2(29)$ & $3(100)$ & $3(75)$ \\
\hline$<30$ days after tracheostomy & $17(45)$ & $0(0)$ & $3(100)$ & $2(50)$ \\
\hline$<90$ days after tracheostomy* & $20(53)$ & $2(29)$ & $3(100)$ & $3(75)$ \\
\hline$<1$ year after tracheostomy* & $20(53)$ & $2(29)$ & $3(100)$ & $3(75)$ \\
\hline Home ventilation & $3(8)$ & $0(0)$ & $0(0)$ & $2(50)$ \\
\hline \multirow[t]{2}{*}{ Variables } & \multicolumn{2}{|l|}{ Tracheostomy } & \multicolumn{2}{|c|}{ Cricothyroidotomy + tracheostomy } \\
\hline & $\begin{array}{l}\text { Trauma } \\
N=38 \\
\text { Median (IQR) }\end{array}$ & $\begin{array}{l}\text { Non-trauma } \\
N=7 \\
\text { Median (IQR) }\end{array}$ & $\begin{array}{l}\text { Trauma } \\
N=3 \\
\text { Median (IQR) }\end{array}$ & $\begin{array}{l}\text { Non-trauma } \\
N=4 \\
\text { Median (IQR) }\end{array}$ \\
\hline Duration weaning period (days) & $2(3)$ & $21 * *$ & $1 * *$ & $1 * *$ \\
\hline Time from trauma/initial surgery to no MV (days) & $15(13)$ & $36 * *$ & $6 * *$ & $4 * *$ \\
\hline Duration decuffing period (days) & $8(6)$ & $19 * *$ & $3 * *$ & $7 * *$ \\
\hline Duration tracheostoma in situ (days) & $19(19)$ & $69 * *$ & $8 * *$ & $15^{* * *}$ \\
\hline Length of hospital stay (days) & $39(17)$ & $66(43)$ & $17 * *$ & $36(46)$ \\
\hline Length of ICU stay (days) & $16(15)$ & $45(47)$ & $6 * *$ & $19 * *$ \\
\hline \multirow[t]{2}{*}{ Variables } & \multicolumn{2}{|l|}{ Tracheostomy } & \multicolumn{2}{|c|}{ Cricothyroidotomy + tracheostomy } \\
\hline & $\begin{array}{l}\text { Trauma } \\
N=38 \\
N(\%)\end{array}$ & $\begin{array}{l}\text { Non-trauma } \\
N=7 \\
N(\%)\end{array}$ & $\begin{array}{l}\text { Trauma } \\
N=3 \\
N(\%)\end{array}$ & $\begin{array}{l}\text { Non-trauma } \\
N=4 \\
N(\%)\end{array}$ \\
\hline \multicolumn{5}{|l|}{ Functional outcomes } \\
\hline Phonation problems & $3(8)$ & $0(0)$ & $0(0)$ & $1(25)$ \\
\hline \multicolumn{5}{|l|}{ Physical airway complaints } \\
\hline Pain/irritation & $3(8)$ & $0(0)$ & $0(0)$ & $1(25)$ \\
\hline Swallowing dysfunction & $7(18)$ & $1(14)$ & $0(0)$ & $0(0)$ \\
\hline Excessive sputum post tracheostomy & $24(63)$ & $1(14)$ & $0(0)$ & $3(75)$ \\
\hline Sputum leakage at tracheostomy site & $9(24)$ & $0(0)$ & $0(0)$ & $1(25)$ \\
\hline Inability to clean airway & $23(61)$ & $2(29)$ & $0(0)$ & $1(25)$ \\
\hline \multicolumn{5}{|l|}{ Material-related outcomes } \\
\hline Material-related complications & $10(32)$ & $0(0)$ & $1(33)$ & $0(0)$ \\
\hline Luxation cannula & $2(5)$ & $0(0)$ & $0(0)$ & $0(0)$ \\
\hline
\end{tabular}


Table 2 continued

\begin{tabular}{|c|c|c|c|c|}
\hline \multirow[t]{4}{*}{ Variables } & \multicolumn{2}{|c|}{ Tracheostomy } & \multicolumn{2}{|c|}{ Cricothyroidotomy + tracheostomy } \\
\hline & Trauma & Non-trauma & Trauma & Non-trauma \\
\hline & $N=38$ & $N=7$ & $N=3$ & $N=4$ \\
\hline & $N(\%)$ & $N(\%)$ & $N(\%)$ & $N(\%)$ \\
\hline Acquired cuff insufficiency & $8(21)$ & $0(0)$ & $0(0)$ & $0(0)$ \\
\hline
\end{tabular}

ICU Intensive care unit, $M V$ mechanical ventilation, IQR Interquartile range

Bold indicates a significant difference $(p \leq 0.05)$ between trauma and non-trauma subgroups.

*Missing data: Data $<90$ days after tracheostomy was missing in 5\% $(n=2)$ of trauma and $14 \%(n=1)$ of non-trauma tracheostomy patients. Data $<1$ year after tracheostomy was missing in $8 \%(n=3)$ of trauma and $14 \%(n=1)$ of non-trauma tracheostomy patients

**In several cases, IQR could not be presented due to group size of $n \leq 3$

of ICU stay, it can be argued that non-trauma patients were more dependent of other ICU utilities besides respiratory support due to underlying disease, elongating their ICU stay. Mehta et al., with a sample size of 18.000 in the US, also showed improved outcomes following tracheostomy in trauma patients compared to non-trauma patients [21].

Although limited in number, cricothyroidotomy did not evidently cause short- or long-term complications-remarkable, since this is always done in urgent, life-threatening situations. This contradicts previous studies reporting several complications following cricothyroidotomy (e.g., cartilage injury, subglottic stenosis and phonation problems) $[16,22,23]$. Focusing on indications for cricothyroidotomy, impossibility to intubate due to facial/airway injuries is reported as one of the major indications 24 . In our study, only one patient underwent cricothyroidotomy due to facial injuries. With approximately 350 polytrauma patients in our center per year, this relates to one cricothyroidotomy due to facial injuries per 1,750 polytrauma patients over a five-year period [25]. Thus, in a mature trauma center, cricothyroidotomy is becoming extremely rare in the ER and is more often performed outside the ER or in non-trauma settings.

Regarding complications following tracheostomy, only pneumonia was frequently present, with similar rates as reported by previous studies $[13,26]$. No other procedural complications occurred, demonstrating the safety of tracheostomy as an airway patency management procedure, even in presumed complex patients. Material-related complications and adverse functional outcomes were observed more frequently, especially in trauma patients, which is in line with the recent literature [27]. However, as also shown by Silvester et al., trauma-related characteristics (such as injuries sustained during trauma or neck immobilization) may have influenced this Silvester et al.
[28]. It can also be argued that trauma patients were more alert to these adverse functional outcomes, since they generally recovered more quickly. In our study, cervical spinal cord injuries significantly increased phonation problems, inability to clean airway and need for home ventilation support. The relation between excessive sputum production pre-tracheostomy and inability to clean airway (reduced airway hygiene) after tracheostomy indicates the problem was already present before tracheostomy and was possibly even part of its indication.

Evaluation of procedural specifics showed an association between a longitudinal incision and increased sputum leakage at tracheostomy site. Although sputum leakage was only significantly associated with a longitudinal incision, our results suggest a combination of cervical injury, early tracheostomy and a longitudinal incision was most likely to cause sputum leakage. This may be explained by local swelling of the incision site at time of tracheostomy, with sputum leakage occurring after reduction in swelling. Caregivers should consider variables such as injury type (e.g., cervical (spinal cord) injury with regional swelling), timing of tracheostomy and incision type when applying surgical airway management care.

\section{Strengths and limitations}

This study provided a detailed description of tracheostomy outcomes-including functional and daily tracheostomy care-related outcomes-in a time where this procedure is predominantly reserved as a back-up procedure. Furthermore, these results could be related to patient, injury/disease and procedural characteristics, thus enhancing specificity. 
Table 3 Evaluation of patient/injury/disease/procedural characteristics and functional outcomes

\begin{tabular}{|c|c|c|c|c|c|c|c|c|c|c|}
\hline & \multicolumn{2}{|c|}{$\begin{array}{l}\text { Phonation problems } \\
N=4\end{array}$} & \multicolumn{2}{|c|}{$\begin{array}{l}\text { Physical airway } \\
\text { complaints } N=12\end{array}$} & \multicolumn{2}{|c|}{$\begin{array}{l}\text { Excessive sputum } \\
\text { post-tracheostomy } \\
N=28\end{array}$} & \multicolumn{2}{|c|}{$\begin{array}{l}\text { Sputum leakage } \\
N=10\end{array}$} & \multicolumn{2}{|c|}{$\begin{array}{l}\text { Inability to clean } \\
\text { airway } \\
N=26\end{array}$} \\
\hline & $\begin{array}{l}\text { Trauma } \\
N=3 \\
(75 \%)\end{array}$ & $\begin{array}{l}\text { Non- } \\
\text { trauma } \\
N=1 \\
(25 \%)\end{array}$ & $\begin{array}{l}\text { Trauma } \\
N=10 \\
(83 \%)\end{array}$ & $\begin{array}{l}\text { Non- } \\
\text { trauma } \\
N=2 \\
(17 \%)\end{array}$ & $\begin{array}{l}\text { Trauma } \\
N=24 \\
(86 \%)\end{array}$ & $\begin{array}{l}\text { Non- } \\
\text { trauma } \\
N=4 \\
(14 \%)\end{array}$ & $\begin{array}{l}\text { Trauma } \\
N=9 \\
(90 \%)\end{array}$ & $\begin{array}{l}\text { Non- } \\
\text { trauma } \\
N=1 \\
(10 \%)\end{array}$ & $\begin{array}{l}\text { Trauma } \\
N=23 \\
(88 \%)\end{array}$ & $\begin{array}{l}\text { Non- } \\
\text { trauma } \\
N=3 \\
(11 \%)\end{array}$ \\
\hline & $N(\%)$ & $N(\%)$ & $N(\%)$ & $N(\%)$ & $N(\%)$ & $N(\%)$ & $N(\%)$ & $N(\%)$ & $N(\%)$ & $N(\%)$ \\
\hline \multicolumn{11}{|l|}{ Demographics } \\
\hline Male & $3(75)$ & $0(0)$ & $8(67)$ & $1(8)$ & $16(57)$ & $1(4)$ & $8(80)$ & $0(0)$ & $17(65)$ & $2(8)$ \\
\hline Female & $0(0)$ & $1(25)$ & $2(17)$ & $1(8)$ & $8(29)$ & $3(11)$ & $1(10)$ & $1(10)$ & $6(23$ & $1(4)$ \\
\hline Diabetes & $0(0)$ & $0(0)$ & $0(0)$ & $0(0)$ & $2(7)$ & $0(0)$ & $1(10)$ & $0(0)$ & $1(4)$ & $0(0)$ \\
\hline \multicolumn{11}{|l|}{ ASA score } \\
\hline 1 & $0(0)$ & $0(0)$ & $0(0)$ & $0(0)$ & $0(0)$ & $0(0)$ & $0(0)$ & $0(0)$ & $0(0)$ & $0(0)$ \\
\hline 2 & $2(50)$ & $0(0)$ & $4(33)$ & $0(0)$ & $13(46)$ & $0(0)$ & $5(50)$ & $0(0)$ & $13(50)$ & $0(0)$ \\
\hline 3 & $1(25)$ & $1(25)$ & $6(50)$ & $2(17)$ & $11(39)$ & $4(4)$ & $4(40)$ & $1(10)$ & $10(38)$ & $2(8)$ \\
\hline 4 & $0(0)$ & $0(0)$ & $0(0)$ & $0(0)$ & $0(0)$ & $0(0)$ & $0(0)$ & $0(0)$ & $0(0)$ & $1(4)$ \\
\hline \multicolumn{11}{|l|}{ Clinical characteristics } \\
\hline GCS motor $\leq 3^{*}$ & $2(50)$ & $0(0)$ & $3(25)$ & $1(8)$ & $12(43)$ & $1(4)$ & $3(30)$ & $1(10)$ & $10(38)$ & $0(0)$ \\
\hline $\mathrm{GCS} \leq 8^{*}$ & $2(50)$ & $0(0)$ & $4(33)$ & $1(8)$ & $14(50)$ & $1(4)$ & $4(40)$ & $1(10)$ & $12(46)$ & $0(0)$ \\
\hline $\begin{array}{l}\text { Spine } \\
\text { immobilisation at } \\
\text { time of } \\
\text { tracheostomy }\end{array}$ & $2(50)$ & $0(0)$ & $8(67)$ & $0(0)$ & $18(64)$ & $0(0)$ & $7(70)$ & $0(0)$ & $18(69)$ & $0(0)$ \\
\hline ICU admission & $0(0)$ & $1(25)$ & $10(83)$ & $1(8)$ & $24(86)$ & $3(11)$ & $9(90)$ & $0(0)$ & $23(82)$ & $3(12)$ \\
\hline $\begin{array}{l}\text { Excessive sputum } \\
\text { production pre- } \\
\text { tracheostomy }\end{array}$ & $1(25)$ & $0(0)$ & $6(50)$ & $0(0)$ & $11(39)$ & $2(50)$ & $4(40)$ & $0(0)$ & $13(50)$ & $2(67)$ \\
\hline \multicolumn{11}{|c|}{ Trauma injury specifics } \\
\hline Head injury & $0(0)$ & $0(0)$ & $6(50)$ & $0(0)$ & $15(54)$ & $0(0)$ & $4(40)$ & $0(0)$ & $13(50)$ & $0(0)$ \\
\hline \multicolumn{11}{|l|}{ Brain injury** } \\
\hline $\begin{array}{l}\text { Mild (GCS motor } \\
4-5)\end{array}$ & $0(0)$ & $0(0)$ & $3(25)$ & $0(0)$ & $7(25)$ & $0(0)$ & $3(30)$ & $0(0)$ & $7(27)$ & $0(0)$ \\
\hline $\begin{array}{l}\text { Severe }(\mathrm{GCS} \\
\text { motor } \leq 3 \text { ) }\end{array}$ & $2(50)$ & $0(0)$ & $3(25)$ & $0(0)$ & $12(43)$ & $0(0)$ & $3(30)$ & $0(0)$ & $10(38)$ & $0(0)$ \\
\hline Cervical injury & $3(75)$ & $0(0)$ & $5(42)$ & $0(0)$ & $13(46)$ & $0(0)$ & $6(60)$ & $0(0)$ & $13(50)$ & $0(0)$ \\
\hline \multicolumn{11}{|c|}{ Cervical spinal cord injury } \\
\hline Level C1-C4 & $2(50)$ & $0(0)$ & $1(8)$ & $0(0)$ & $3(11)$ & $0(0)$ & $2(20)$ & $0(0)$ & $5(19)$ & $0(0)$ \\
\hline Level C5-C8 & $0(0)$ & $0(0)$ & $1(8)$ & $0(0)$ & $2(7)$ & $0(0)$ & $0(0)$ & $0(0)$ & $2(8)$ & $0(0)$ \\
\hline ISS $>15$ & $3(75)$ & - & $8(67)$ & - & $19(68)$ & - & $8(80)$ & - & $2(8)$ & - \\
\hline \multicolumn{11}{|l|}{ Procedural specifics } \\
\hline \multicolumn{11}{|c|}{ Time to tracheostomy } \\
\hline Early ( $\leq 14$ days) & $3(75)$ & $1(25)$ & $8(67)$ & $1(8)$ & $17(61)$ & $4(14)$ & $8(80)$ & $1(10)$ & $21(81)$ & $2(8)$ \\
\hline Late $(>14$ days $)$ & $0(0)$ & $0(0)$ & $2(17)$ & $1(8)$ & $7(25)$ & $0(0)$ & $1(10)$ & $0(0)$ & $2(8)$ & $1(4)$ \\
\hline \multicolumn{11}{|l|}{ Indication } \\
\hline $\begin{array}{l}\text { Physically } \\
\text { threatened airway }\end{array}$ & $0(0)$ & $1(25)$ & $0(0)$ & $0(0)$ & $1(4)$ & $1(4)$ & $1(10)$ & $0(0)$ & $1(4)$ & $1(4)$ \\
\hline $\begin{array}{l}\text { Insufficient } \\
\text { respiratory } \\
\text { function }\end{array}$ & $3(75)$ & $0(0)$ & $9(75)$ & $1(8)$ & $22(79)$ & $1(4)$ & $8(80)$ & $0(0)$ & $22(85)$ & $2(8)$ \\
\hline $\begin{array}{l}\text { Alternative way of } \\
\text { airway access }\end{array}$ & $0(0)$ & $0(0)$ & $1(8)$ & $1(8$ & $1(4)$ & $2(7)$ & $0(0)$ & $1(10)$ & $0(0)$ & $0(0)$ \\
\hline
\end{tabular}


Table 3 continued

\begin{tabular}{|c|c|c|c|c|c|c|c|c|c|c|}
\hline & \multicolumn{2}{|c|}{$\begin{array}{l}\text { Phonation problems } \\
N=4\end{array}$} & \multicolumn{2}{|c|}{$\begin{array}{l}\text { Physical airway } \\
\text { complaints } N=12\end{array}$} & \multicolumn{2}{|c|}{$\begin{array}{l}\text { Excessive sputum } \\
\text { post-tracheostomy } \\
N=28\end{array}$} & \multicolumn{2}{|c|}{$\begin{array}{l}\text { Sputum leakage } \\
N=10\end{array}$} & \multicolumn{2}{|c|}{$\begin{array}{l}\text { Inability to clean } \\
\text { airway } \\
N=26\end{array}$} \\
\hline & $\begin{array}{l}\text { Trauma } \\
N=3 \\
(75 \%)\end{array}$ & $\begin{array}{l}\text { Non- } \\
\text { trauma } \\
N=1 \\
(25 \%)\end{array}$ & $\begin{array}{l}\text { Trauma } \\
N=10 \\
(83 \%)\end{array}$ & $\begin{array}{l}\text { Non- } \\
\text { trauma } \\
N=2 \\
(17 \%)\end{array}$ & $\begin{array}{l}\text { Trauma } \\
N=24 \\
(86 \%)\end{array}$ & $\begin{array}{l}\text { Non- } \\
\text { trauma } \\
N=4 \\
(14 \%)\end{array}$ & $\begin{array}{l}\text { Trauma } \\
N=9 \\
(90 \%)\end{array}$ & $\begin{array}{l}\text { Non- } \\
\text { trauma } \\
N=1 \\
(10 \%)\end{array}$ & $\begin{array}{l}\text { Trauma } \\
N=23 \\
(88 \%)\end{array}$ & $\begin{array}{l}\text { Non- } \\
\text { trauma } \\
N=3 \\
(11 \%)\end{array}$ \\
\hline & $N(\%)$ & $N(\%)$ & $N(\%)$ & $N(\%)$ & $N(\%)$ & $N(\%)$ & $N(\%)$ & $N(\%)$ & $N(\%)$ & $N(\%)$ \\
\hline \multicolumn{11}{|l|}{ Incision type } \\
\hline Longitudinal & $3(75)$ & $0(0)$ & $7(58)$ & $1(8)$ & $19(68)$ & $1(4)$ & $9(90)$ & $1(10)$ & $7(27)$ & $0(0)$ \\
\hline U-shaped & $0(0)$ & $1(25)$ & $3(25)$ & $0(0)$ & $5(18)$ & $2(7)$ & O (0) & $0(0)$ & $7(27)$ & $2(8)$ \\
\hline $\begin{array}{l}\text { Cricothyroidotomy } \\
\text { pre-tracheostomy }\end{array}$ & $0(0)$ & $1(25)$ & $0(0)$ & $1(8)$ & $0(0)$ & $3(11)$ & $0(0)$ & $1(10)$ & $0(0)$ & $1(4)$ \\
\hline
\end{tabular}

ASA American society of anesthesiologists, GCS Glasgow coma scale

Bold indicates a significant relation between variables with $p \leq 0.05$

*Missing data: GCS was missing in one non-trauma patient, incision type was missing in one non-trauma patient

**Bain injury was scored as mild if GCS motor $3-5$, and scored as severe if GCS motor $\leq 3$

Our study was limited by a relatively small sample size and subsequently, the inability to perform adequate multivariate analysis to control for potential confounders. The small sample size of surgical tracheostomy technique in emergency surgery patients was primarily caused by the increasing rarity of tracheostomy procedures and the recent shift to the percutaneous tracheostomy technique in current medical practice. Another limitation was its retrospective nature. Furthermore, in our study, no validated patient reported outcome measures were used to analyze functional outcomes. Finally, outcomes for patients who were spared tracheostomy by postponing the procedure were not accounted for. These outcomes could affect decisionmaking regarding both indication and timing.

\section{Conclusion}

In an era in which surgical airway management has become a back-up procedure and is reserved for complex patients only, a detailed description of clinical results is scarce. In our study, all patients had either a cervical or systemic problem, which posed a contra-indication for percutaneous procedures. Nevertheless, no major procedure-related complications or functional adverse events were encountered; only temporary functional complications occurred. Our results show that trauma patients with cervical injury, who underwent early tracheostomy with a longitudinal incision, were especially at risk of these temporary complications. This information can aid clinicians in making tailor-made decisions for individual patients.

Author contributions All authors have made substantial contributions to the interpretation of data, drafting and/or revising the manuscript and gave final approval for submission.

\section{Funding None.}

Data availability The datasets used and/or analyzed during the current study are available from the corresponding author on reasonable request.

\section{Declarations}

Conflict of interest The authors declare that they have no competing interest.

Ethical approval A waiver was provided by the institutional review board of the University Medical Center Utrecht.

Consent to participate A waiver was provided by the institutional review board of the University Medical Center Utrecht.

Open Access This article is licensed under a Creative Commons Attribution 4.0 International License, which permits use, sharing, adaptation, distribution and reproduction in any medium or format, as long as you give appropriate credit to the original author(s) and the source, provide a link to the Creative Commons licence, and indicate if changes were made. The images or other third party material in this article are included in the article's Creative Commons licence, unless indicated otherwise in a credit line to the material. If material is not 
included in the article's Creative Commons licence and your intended use is not permitted by statutory regulation or exceeds the permitted use, you will need to obtain permission directly from the copyright holder. To view a copy of this licence, visit http://creativecommons. org/licenses/by/4.0/.

\section{References}

1. McGill J, Clinton JE, Ruiz E (1982) Cricothyrotomy in the emergency department. Ann Emerg Med 11(7):361-364

2. Johnson DR, Dunlap A, McFeeley P et al (1993) Cricothyrotomy performed by prehospital personnel: a comparison of two techniques in a human cadaver model. Am J Emerg Med 11(3):207-209

3. McCredie VA, Alali AS, Scales DC et al (2017) Effect of early versus late tracheostomy or prolonged intubation in critically Ill patients with acute brain injury: a systematic review and metaanalysis. Neurocrit Care 26(1):14-25

4. Adly A, Youssef TA, El-Begermy MM et al (2018) Timing of tracheostomy in patients with prolonged endotracheal intubation: a systematic review. Eur Arch Otorhinolaryngol 275(3):679-690

5. Rodriguez JL, Steinberg SM, Luchetti FA et al (1990) Early tracheostomy for primary airway management in the surgical critical care setting. Surgery 108:65-69

6. Whited RE (1984) A prospective study of laryngotracheal sequelae in long-term intubation. Laryngoscope 94:367-377

7. Heffner JE, Hess D (2001) Tracheostomy management in the chronically ventilated patient. Clin Chest Med 22(1):55-69

8. Nieszkowska A, Combes A, Luyt CE et al (2005) Impact of tracheostomy on sedative administration, sedation level, and comfort of mechanically ventilated intensive care unit patients. Crit Care Med 33(11):2527-2533

9. Freeman BD (2017) Tracheostomy update: when and how. Crit Care Clin 33(2):311-322

10. Lin WC, Chen CW, Wang JD et al (2015) Is tracheostomy a better choice than translaryngeal intubation for critically ill patients requiring for more than 14 days? a comparison of shortterm outcomes. BMC Anesthesiol 15:181

11. Groves DS, Durbin CG Jr (2007) Tracheostomy in the critically ill: indications, timing and techniques. Curr Opin Crit Care 13(1):90-97

12. Dempsey GA, Morton B, Hammell C et al (2016) Long-term outcome following tracheostomy in critical care: a systematic review. Crit Care Med 44(3):617-628

13. Clec'h C, Alberti C, Vincent F et al (2007) Tracheostomy does not improve the outcome of patients requiring prolonged mechanical ventilation: a propensity analysis. Crit Care Med 35(1):132-138
14. Plate JDJ, Leenen LPH, Houwert M et al (2017) Utilisation of intermediate care units: a systematic review. Crit Care Res Pract 2017:8038460

15. Minei JP, Brakenridge SC, Phelan HA (2011) Outcomes and long term follow-up after emergent cricothyroidotomy: is routine conversion to tracheostomy necessary? Am Surg 77(12):1707-1711

16. Talving P, DuBose J, Inaba K et al (2010) Conversion of emergent cricothyrotomy to tracheostomy in trauma patients. Arch Surg 145(1):87-91

17. Patel SA, Plowman EK, Halum S et al (2015) Late tracheostomy is associated with higher morbidity and mortality in mechanically ventilated patients. Laryngoscope 125(9):2134-2138

18. Rumbak MJ, Newton M, Truncale T et al (2004) A prospective, randomized, study comparing early percutaneous dilational tracheostomy to prolonged translaryngeal intubation (delayed tracheostomy) in critically ill medical patients. Crit Care Med 32(8):1689-1694

19. Frutos-Vivar Fernando, Esteban Andres et al (2005) Outcome of mechanically ventilated patients who require a tracheostomy. Crit Care Med. 33(2):290-298

20. Shah RK, Lander L, Berry JG et al (2012) Tracheostomy outcomes and complications: a national perspective. Laryngoscope 122(1):25-29

21. Mehta AB, Cooke CR, Wiener RS et al (2016) Hospital variation in early tracheostomy in the united states: a population-based study. Crit Care Med 44(8):1506-1514

22. Gillespie MB, Eisele DW (1999) Outcomes of emergency surgical airway procedures in a hospital-wide setting. Laryngoscope 109(11):1766-1769

23. Bair AE, Panacek EA, Wisner DH et al (2003) Cricothyrotomy: a 5-year experience at one institution. J Emerg Med 24:151-156

24. Hart KL, Thompson SH (2010) Emergency cricothyrotomy. Atlas Oral Maxillofac Surg Clin North Am 18(1):29-38

25. Voskens FJ, van Rein EAJ, van der Sluijs R et al (2018) Accuracy of prehospital triage in selecting severely injured trauma patients. JAMA Surg 153(4):322-327

26. Keenan JE, Gulack BC, Nussbaum DP et al (2015) Optimal timing of tracheostomy after trauma without associated head injury. J Surg Res 198(2):475-481

27. Klotz R, Probst P, Deininger M et al (2018) Percutaneous versus surgical strategy for tracheostomy: a systematic review and metaanalysis of perioperative and postoperative complications. Langenbecks Arch Surg 403(2):137-149

28. Silvester W, Goldsmith D, Uchino S et al (2006) Percutaneous versus surgical tracheostomy: a randomized controlled study with long-term follow-up. Crit Care Med 34(8):2145-2152

Publisher's Note Springer Nature remains neutral with regard to jurisdictional claims in published maps and institutional affiliations. 